\title{
Comparative study between duplex $\&$ angiographic guided angioplasty for failing arterio-venous fistulas
}

\author{
Hisham Moustafa, ${ }^{a} M D ;$ Essam Balegh, ${ }^{b} M D ;$ Hatem Elazizi, ${ }^{c} M D$; \\ Khaled Elkaffas, ${ }^{c} M D$
}

\author{
a) Department of General Surgery, Cairo University, Cairo, Egypt. \\ b) Department of Cardiology, Cairo University, Cairo, Egypt. \\ c) Department of Radio-diagnosis, Cairo University, Cairo, Egypt.
}

\begin{abstract}
Introduction: There was no existing consensus regarding the optimal treatment for the failing arterio-venous fistulas. Compared with surgery, percutaneous trans-luminal angioplasty (PTA) has several advantages, including simplicity, minimal invasiveness, shorter time and less stress to the patients. It also enables immediate dialysis without the need for central venous catheter, reduces the risk of infection, and saves the patient's veins. ${ }^{6}$ PTA can be done angiographic or duplex guided. The rationale for duplex guidance is the avoidance of both radiation exposure and contrast reactions.

Aim of the study: The aim of this study is to elaborate and evaluate the technique of duplex guided balloon angioplasty for failing AVF in Kasr Elaini Hospitals and to compare it with the angiographic guidance.

Patients \& methods: This study included 68 patients with failing hemodialysis AVFs (38 males and 30 females). After having informed written consent, every patient was subjected to history taking, physical examination and duplex evaluation. The patients were randomly allocated between two groups of management to have 34 patients in each group. These groups are the duplex guided group (A) and the angiographic guided group (B). The merits and limitations for duplex and angiographic guidance were compared.

Results: Duplex guidance obviates the need for contrast injection, radiation exposure and angio-suite. It is a real time and gives an idea about the hemodynamics and is of less cost when compared with angiographic guidance. On the other hand, angiographic guidance is panoramic with better central veins delineation and with significantly shorter duration (ranged from 25 to $50 \mathrm{~min}$ with a mean of $36 \mathrm{~min} \pm 12$ ). The duplex guided balloon angioplasty duration ranged from 35 to 110 min (mean $45 \mathrm{~min} \pm 18$ ) (P value <0.05).

Conclusion: Balloon angioplasty for failing AVF is an excellent promising modality for fistula salvage whether done under angiographic or duplex guidance with merits for each.
\end{abstract}

\section{Introduction:}

End stage renal disease (ESRD) patients on hemodialysis outnumber those on peritoneal dialysis or transplantation, ${ }^{1,2}$ so the creation and maintenance of vascular access for hemodialysis is crucial. Vascular access should be easy to access, safe, effective and durable..$^{3,4}$ However, there is no vascular access for hemodialysis that satisfies all these requirements forever. So ESRD patients usually require several procedures and/or interventions if the original access is failing. Failing arterio- venous fistula is defined as one with more than $50 \%$ reduction in the normal vessel caliber associated with hemodynamic, functional, or clinical abnormality. 5

There was no existing consensus regarding the optimal treatment for the failing arteriovenous fistulas. Compared with surgery, percutaneous transluminal angioplasty (PTA) has several advantages, including simplicity, minimal invasiveness, shorter time and less stress to the patients. It also enables immediate dialysis without the need for central venous 
catheter, reduces the risk of infection, and saves the patient's veins. ${ }^{6}$ PTA can be done angiographic or duplex guided. The rationale for duplex guidance is the avoidance of both radiation exposure and contrast reactions.

\section{Aim of the study:}

The aim of this study is to elaborate and evaluate the technique of duplex guided balloon angioplasty for failing AVF in Kasr Elaini Hospitals and to compare it with the angiographic guidance.

\section{Patients and methods:}

This study included 68 patients with failing hemodialysis AVFs (38 males and 30 females). The patients were either on regular hemodialysis (52 patients) or prepared for it (16 patients). Failing access was documented clinically or by duplex scanning. The inclusion and exclusion criteria are included in Table(1).

\section{Table (1): Inclusion and exclusion criteria.}

\begin{tabular}{|l|}
\hline Inclusion criteria: \\
\hline - Reduction of palpable thrill, \\
\hline - Repeated problematic cannulation, \\
\hline - Decreased flow rate and variable pump speed, \\
\hline - Under dialysis or prolonged period of hemodialysis > 4 hours, \\
\hline - Frequent withdrawal of blood clots, \\
\hline - Prolonged bleeding after cannulation, \\
\hline - Stenosis causing > 50\% reduction in the luminal vessel diameter by duplex scanning. \\
\hline Exclusion criteria: \\
\hline - Infection. \\
\hline - Systemic low flow status. \\
\hline - External compression by hematoma. \\
\hline
\end{tabular}

After an informed written consent, patients were subjected to history taking with special emphasis on age, gender, cause of renal failure, co-morbidities as well as current and prior dialysis access. The upper limbs were examined for scars, edema, dilated veins, aneurysmal dilatations, thrombosis, hematomas, prominent pulsations and thrill propagation.

The study patients were pre-operatively evaluated by 5-7 MHz linear array duplex transducer of the available duplex machine (Philips EnVisor or Semines Eligera) in both longitudinal and transverse planes by well trained operator focusing on the following essential items that guide the management plan:

- The number and site of stenotic segments in the arterial part, venous part and/or the site of anastomosis itself. Accordingly stenosis was classified into arterial proximal, arterial juxta anastomotic, anastomotic, venous perianastomotic, needling segment, or central vein.

- The severity and length of each stenosis.

- The caliber of the vessel proximal and distal to the stenosis.

- The peak systolic velocity (PSV) at the stenotic segment.

- The PSV ratio (PSV at the stenosis/ PSV proximal).

- The flow rate (mean systolic velocity $\mathrm{x}$ vessel area).

- The suitable puncture site for endovascular intervention regarding caliber, patency and continuation. (Sometimes the radial artery had high brachial origin well above the level of brachio-cephalic anastomosis).

- The contribution of the ulnar and radial arteries in hand circulation to justify the safety of using either of them as a puncture site. 
The 16 patients who didn't start dialysis yet were scheduled for duplex guided balloon angioplasty. However, the rest of the patients were randomly allocated between two groups of management to have finally 34 patients in each group. The first group (group A) was subjected to duplex guided angioplasty and the second (group B) was subjected to angiography guided angioplasty. The study was approved by the ethics review board of the hospitals.

\section{Duplex guided balloon angioplasty for failing AVF (group A):}

Immediately, prior to the procedure the AVF was re-evaluated and the puncture site was determined. The settings were adjusted regarding the position of the patient, the duplex machine, the duplex operator, the surgeon, the assistant and the scrub nurse Figure(1). The field was sterilized and a linear $5-7 \mathrm{MHz}$ probe inserted in a sterile plastic cover and sterile gel were put in the field. The patients were monitored clinically, and by pulse oximetry and ECG all-through the procedure. The procedures were performed under local anesthesia at the puncture site (2\% lidocaine hydrochloride) and light sedation during balloon inflation. The distal part of the radial artery was the puncture site of choice in most of the cases as it can tackle both the artery and the vein and can be controlled post-procedural without hindering the fistula flow.

The radial artery was punctured percutaneously or through surgical cut-down direct exposure technique with a $30-\mathrm{mm} 20$ $\mathrm{G}$ sheathed or unsheathed needle. The needle was exchanged over a short wire for a $5-\mathrm{Fr}$ radial sheath. Heparin (5000IU) was given to prevent intra-procedural thrombosis. A $5 \mathrm{Fr}$ selective Bernstein catheter (Boston Sceintefic) was introduced over 0.035 " hydrophilic guidewire through the sheath under duplex guidance Figures $(2,3)$. The wire and the catheter were then manipulated and advanced under B-mode guidance aided by external manual venous or arterial compression until the wire was advanced and secured across the lesion. The Bernstein catheter was exchanged for a peripheral balloon Figure(4). The balloon size and length were determined according to duplex measurements of the size of the vein adjacent to the area to be dilated and the length of the lesion. The balloons used ranged from $3 \mathrm{~mm}$ to $6 \mathrm{~mm}$ diameter size and from $2 \mathrm{~cm}$ to $6 \mathrm{~cm}$ length. The balloon was inflated for 30 seconds each time. Evaluation of the adequacy of PTA was done morphologically using the B mode for identification of residual stenosis or recoils and hemodynamically using spectral analyses including PSV, PSV ratio and blood flow.

\section{Angiographic guided balloon angioplasty (group B):}

This was done in angio-suite in a conventional way.

Procedural time was measured from the start of puncture to completion of the endovascular procedure. The procedure time did not include the time to achieve haemostasis of the puncture site. Technical success was defined as restoration of flow with residual stenosis of less than $30 \%$ and PSV ratio less than 2. Clinical success was defined as the ability to carry out hemodialysis for at least one week via the treated AVF. Primary patency (PP) rate was defined as the period between the intervention and fistula thrombosis, repeated intervention or abundance of the fistula (in patients who received renal transplantation).

The merits and limitations for duplex and angiographic guidance were compared in both study groups and the patients were scheduled to come for follow up visits including a physical examination and duplex scan within a week and month after the procedure and then every 3 months thereafter for 18 months. 


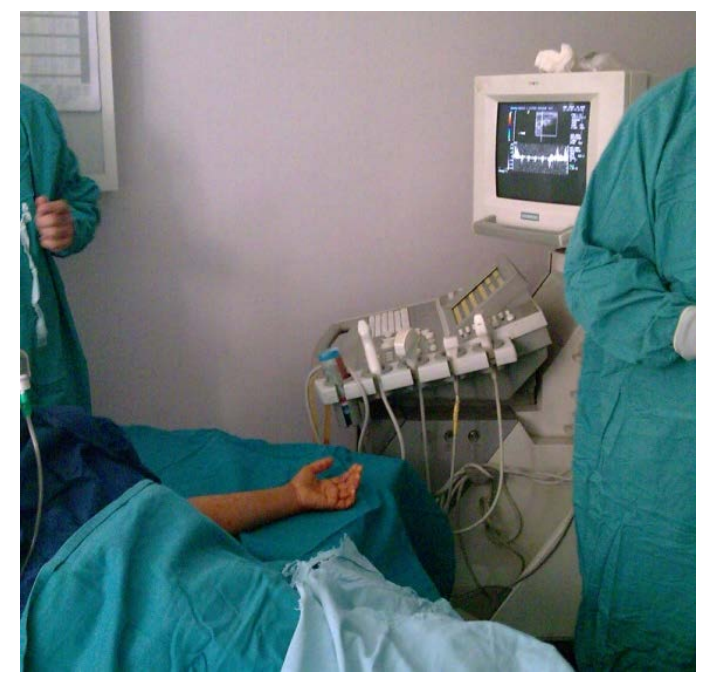

Figure (1): Setting for duplex guided angioplasty.

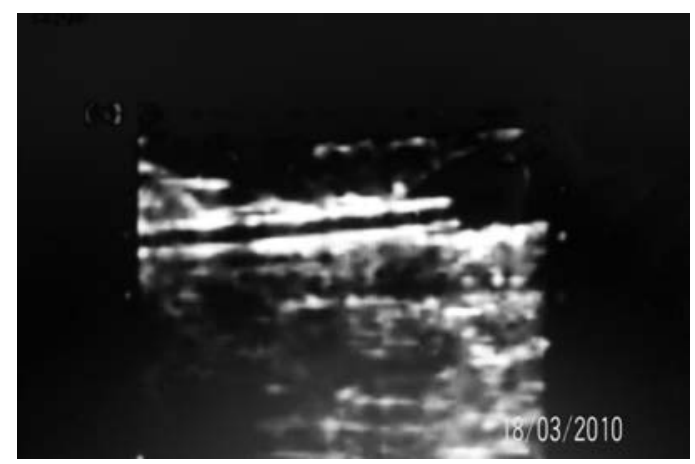

Figure (3): The Bern catheter tip in the vein ready for the passage of the wire.

\section{Results:}

The mean age for the studied patients was $51.8 \pm 14$ years. Diabetes mellitus was responsible for the renal disease in 29 patients $(42.6 \%)$ and chronic glomerulo-nephritis in 15 patients $(22 \%)$. History of multiple previous access surgery was encountered in 41 patients

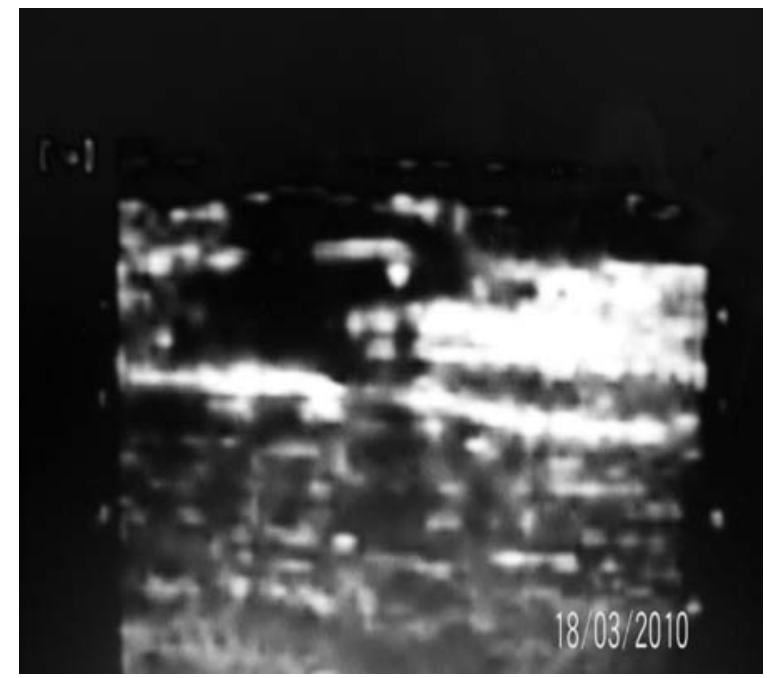

Figure (2): The J-shaped tip of the guidewire entering the vein.

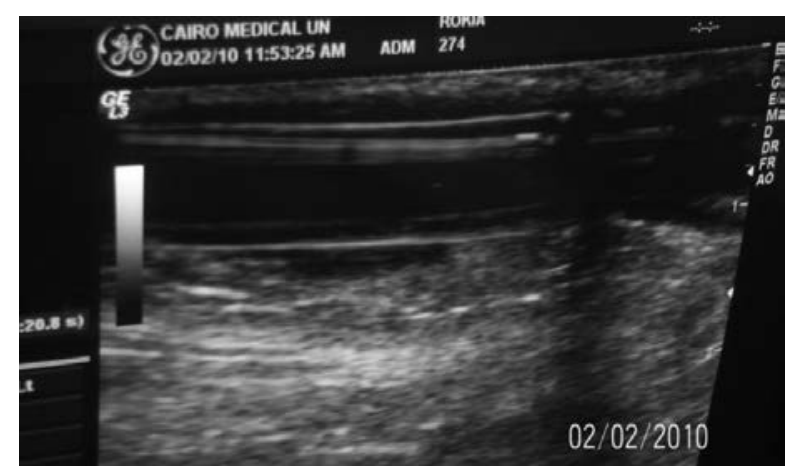

Figure (4): The balloon with the distal metallic mark casting posterior shadow.

$(60.3 \%)$ and the mean duration of the fistulas since their construction was $32 \pm 9.5 \%$ months. Both groups A \& B were well matched regarding age, sex, co-morbidities, history of previous fistula, type $\&$ time of current fistula and the number and site of the stenosis Table(2). 
Table (2): Pre intervention parameters in both groups.

\begin{tabular}{|l|c|c|c|}
\hline & Group A & Group B & P-value \\
\hline Age & $50 \pm 17 \mathrm{y}$ & $53 \pm 12 \mathrm{y}$ & $>0.05$ \\
\hline Male & 17 & 21 & $>0.05$ \\
\hline Diabetes mellitus & 14 & 15 & $>0.05$ \\
\hline History of previous fistula & 20 & 21 & $>0.05$ \\
\hline Sites of the current fistulae & & & $>0.05$ \\
$\quad$ Radiocephalic & 19 & 17 & \\
$\quad$ Brachiocephalic & 12 & 13 & \\
$\quad$ Brachiobasilic & $30 \pm 12 \mathrm{~m}$ & $34 \pm 17 \mathrm{~m}$ & $>0.05$ \\
\hline Time from constructing the fistula & $1-3$ & $1-3$ & $>0.05$ \\
\hline Number of significant stenosis per patient & 47 & & \\
\hline Nu of venous sites of significant stenosis & 34 & 33 & \\
\hline Adjacent to the fistula & 10 & 9 & \\
\hline At the needle site & 3 & 4 & \\
\hline Distal to the needle site & & 4 & \\
\hline
\end{tabular}

The parameters confirming significantly failing fistulas were found to be the followings: diameter narrowing of $50 \%$ or more at grayscale imaging, peak systolic velocity greater than $375 \mathrm{~cm} / \mathrm{s}$, PSV ratio more than 3 , or blood flow $<300 \mathrm{ml} / \mathrm{min}$.

A single stenosis was seen in 45 patients $(66.17 \%)$ and multiple stenoses were found in 23 patients $(33.83 \%)$. The majority of the stenoses were located in the outflow veins mostly juxta-anastomotic Figure(5). The stenosis severity ranged from 50 to $90 \%$, and their lengths ranged from 4 to $30 \mathrm{~mm}$. The PSV ranged from 250 to $700 \mathrm{~cm} / \mathrm{sec}$ and the PSV ratio reached or exceeded 3 . The calculated access blood flow rates were less than $300 \mathrm{ml} / \mathrm{min}$ in all the cases.

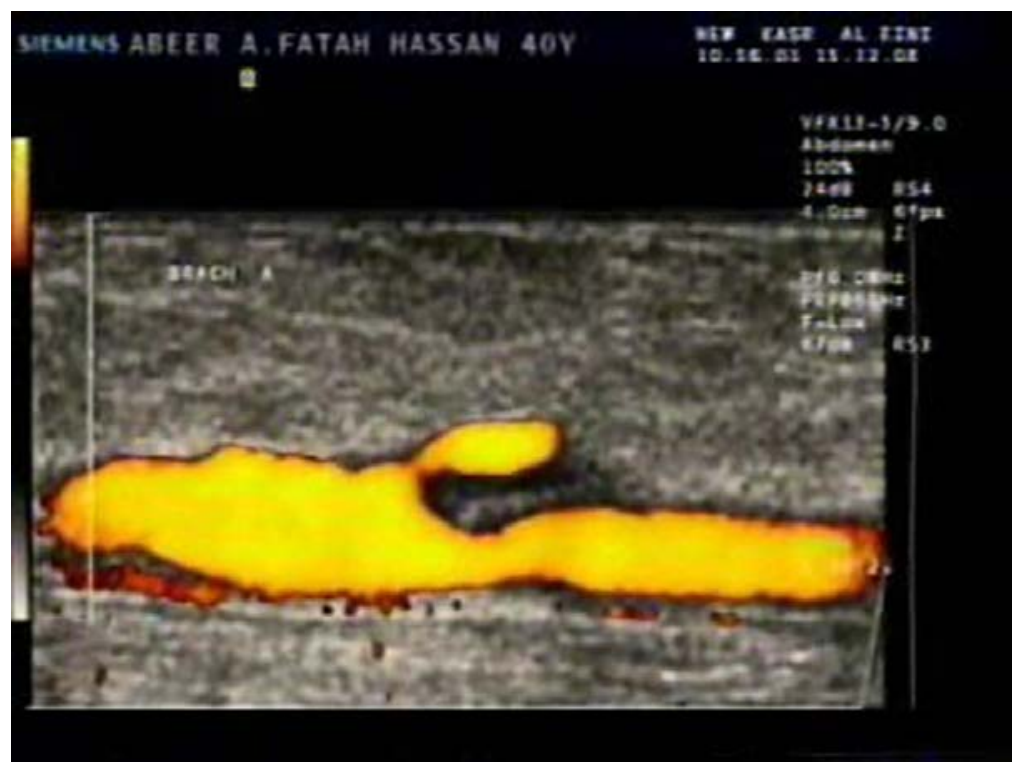

Figure (5): Tight juxta-anastomotic venous stenosis. 
Comparing the techniques in both groups yielded the following advantages for each of them:

Duplex guidance advantages (group A):

- It obviates the need for contrast injection, hence its value for those having allergy to the contrast media, or prepared for but not starting dialysis yet.

- It obviates the need for radiation exposure, hence its safety during pregnancy and for the medical team.

- It obviates the need for angio-suite which may not be available everywhere.
- It is a real time, delineating the fistula lumen and the stenotic areas during wire, catheter and balloon manipulation, unlike the angiographic guidance which can only give a silhouette if the road-map character is evoked.

- It does not only give an idea about the morphology, but far more importantly it gives a good idea about the hemodynamics for better detection of significant residual stenosis.

- It is of less cost when compared with angiographic guidance Table(3).

Table (3): Cost analysis of both procedures in LE (excluding the fees of the personnel).

\begin{tabular}{|l|c|c|}
\hline & Duplex guided (A) & Angiographic guided (B) \\
\hline Place & $200-400 \mathrm{LE}$ & $1500-2000 \mathrm{LE}$ \\
\hline Material & $1500-2500 \mathrm{LE}$ & $1500-2500 \mathrm{LE}$ \\
\hline Contrast & - & $300 \mathrm{LE}$ \\
\hline Total & $1700-2900 \mathrm{LE}$ & $3300-4800 \mathrm{LE}$ \\
\hline
\end{tabular}

Angiographic guidance advantages (group B):

- It is panoramic delineating a considerable length of the access at a time whereas the duplex delineates segment by segment.

- The central veins are better delineated.

- It is notdependent upon a professional duplex operator.

- The angiographic guided PTA, duration is less (ranged from 25 to 50 min with a mean of $36 \mathrm{~min} \pm 12$ ). The duplex guided balloon angioplasty duration ranged from 35 to 110 min (mean $45 \min \pm 18$ ).

The technical success in the duplex guided group was $79.4 \%$ (27 patients). Technical failures were encountered in 7 cases in the early phase of this work. One of them was due to difficult access from the start; one was attributed to failure of traversing the stenotic lesion by the guide-wire due to the presence of extensive intra-venous synachiae at the needling segment; the third one was attributed to rupture of the vein at the angioplasty site during balloon inflation. The remaining four were attributed to resilience, recoil and early re-stenosis $>30 \%$. Despite residual stenosis of more than $30 \%$, three were capable to provide adequate dialysis, so the clinical success was $88 \%$ (30 patients). The peak systolic velocity ratio which was 3 or more before PTA dropped below 2 down to one in all successful cases. Significant improvement in access flow was declared by finding good dancing pulsations in the venous limb post the stenotic area. The technical success in angiographic guidance group occurred in 31 patients $91 \%$.

AVFs that were successfully salvaged by duplex guidance or angiographic guidance were used for hemodialysis the $2^{\text {nd }}$ day after the procedure and there was no need for temporary hemodialysis catheters. The primary patency (PP) rates for the salvaged AVFs under duplex guidance at $1,3,6,9,12,15$ and 18 months were $100 \%, 92 \%, 84 \%, 73 \%, 65 \%$, $50 \%$ and $38 \%$, respectively. The primary patency rates of the salvaged AVFs under angiographic guidance at 1, 3, 6, 9, 12, 15 and 18 months were $100 \%, 100 \%, 89 \%, 78 \%, 55 \%$, $44 \%$ and $33 \%$, respectively.

Compromised AVF patency during the follow up period included those subjected to thrombosis or re-stenosis. Patients lost to follow up were also regarded as compromised patency based on the worst case scenario. Patients who 
underwent transplantation with fistula closure were also included.

\section{Discussion:}

It has been said that "insanity is doing the same thing, in the same way, and expecting a different result" (Roger Milliken). This applies to the research work concerned with hemodialysis access. We can no longer continue doing the same thing (very little indeed), the same way (tabulating data on small scale studies), and expect that the lives of our ESRD patients will be better or that the costs of access care will diminish. It is time to change the paradigm to work proactively and cooperatively to improve the outcomes of hemodialysis access care. Beginnings are half of the journey.

Although arterio-venous fistula is the best available form of hemodialysis access, yet a significant number of fistulas never mature to support dialysis (early failure) or fail after successful use (late failure). ${ }^{7}$ In the past when confronted with such failing fistulas, the usual response was to forget this access completely, put a temporary catheter and plan for a new access. However, based on the limited number of access sites available for each patient and the increasing life expectancy of patients on hemodialysis, the kidney disease outcome quality initiatives (K/DOQI) guidelines recommend early detection and treatment of all hemodynamically significant fistula stenoses, both to extend the life span of each access and to avoid the need to use a temporary hemodialysis catheter. ${ }^{8}$

There was no existing consensus regarding the optimal option for treating the failing AV access. However, the open, surgical, and endovascular treatments should be viewed as alternative or complementary approaches rather than competitive ones. The excellent duplex imaging quality and the technical advances in the endovascular tools allowed a safe performance of duplex guided balloon angioplasties for failing AVF. ${ }^{9}$ The duplex operator who guides the angioplasty must have extensive experience in duplex mapping of the AV access as well as develops the ability to recognize different endovascular tools and devices on the ultrasound image. During the progress of this study, the whole team became acquainted with the images of different endovascular tools. The guide wire appeared as hyper-echoic dot in TS and line in LS. The catheter appeared as double hyper-echoic lines in LS. The balloon appeared in LS as multiple hyper-echoic lines with well evident 2 hyperechoic marks with posterior acoustic shadow. Duplex guidance allowed real-time imaging and monitoring of the intervention. The anstomotic site can be seen while manipulating the wire and the guiding catheter, whereas in the angiographic guidance this can be done on the silhouette of the road map with strict immobility of the patients arm and the C-arm.

Measurement of the access flow parameters during PTA could be helpful to optimize the procedure results. Simply, the reduction of the PSV ratio after PTA to one or around means that the stenosis is perfectly dilated or became insignificant. In certain cases when the stenoses located adjacent to areas with aneurysmal dilatation, it is important to correct the hemodynamic status rather than to correct the morphology.

In the present study, the technical success rates were comparable to the published studies that reported technical success rates ranging from $76 \%$ to $100 \% .10$ In this study, the primary patency rates were consistent with the outcomes observed by Trumel-Rodrigues et al 2000. ${ }^{11}$ The average procedure time of duplex guided angioplasty was longer than that of angiographic guidance because of the frequent assessment of the fistula hemodynamics after each dilatation. Cost analysis showed that the mean total cost of angiographic guided PTA was higher than the duplex guided PTA, mainly because of the use of contrast agent and the angio suite. One of the limitations of the duplex guided angioplasty of failing hemodialysis fistulas is that the sensitivity of duplex for detecting significant central venous lesions is only $80 \%$ if not less.

\section{Conclusion:}

Balloon angioplasty for failing AVF is an excellent promising modality for fistula salvage whether done under angiographic or duplex guidance with merits for each. 


\section{References:}

1- Offner G, Aschendorff C, Hoyer PF, Krohn HP, Ehrich JH, Pichlmayr R, Brodehl J: End stage renal failure: 14 years' experience of dialysis and renal transplantation. Arch Dis Child 1988; 63(2): 120-126.

2- Van Woerden HC, Wilkinson J, Heaven M, Merrifield J: The effect of gender, age, and geographical location on the incidence and prevalence of renal replacement therapy in Wales. BMC Nephrol 2007; 8: 1.

3- Richard Fluck: Summary of clinical practice guidelines for vascular access for haemodialysis. The Renal Association 2010 Oct 26.

4- Jaeschke R, Guyatt GH, Dellinger P et al: Use of GRADE grid to reach decisions on clinical practice guidelines when consensus is elusive. BMJ 2008; 337: 327-330.

5- Tonelli M, Jindal K, Hirsch D, Taylor S, Kane C, Henbrey S: Screening for subclinical stenosis in native vessel arteriovenous fistulae. J Am Soc Nephrol 2001; 12: 1729-1733.

6- Longwitz D, Pham TH, Heckemann RG, Hecking E: Angioplasty in the stenosed hemodialysis shunt: Experiences with 100 patients and 166 interventions. ROFO 1998; 169(1): 68-76.
7- Mercado C, Salman L, Krishnamurthy G, Choi K, ArtIkov S, Thomas I, Merrill D, Asif A: Early and late fistula failure. Clin Nephrol 2008; 69: 77-83.

8- Ives CL, Acoh JA, George J, VaughanHuxley E, Lawson H: Pre-operative vessel mapping and early post-operative surveillance duplex scanning of arteriovenous fistulae. J Vasc Access 2009; 10: 37-42.

9- Marks N, Hingorani A, Ascher E: New office-based vascular interventions. Perspect Vasc Surg Endovasc Ther 2008; 20: 346-347.

10-Hansen MA, Gibsholm-Madsen K, Christensen T, Ladefoged SD: Endovascular treatment of dysfunctional haemodialysis fistulas. Ugeskr Laeger 2009; 171(1-2): 41-45.

11-Turmel-Rodrigues L, Pengloan J, Baudin S, Testou D, Abaza M, Dahdah G, Mouton A, Blanchard D: Treatment of stenosis and thrombosis in hemodialysis fistulas and grafts by interventional radiology. Nephrol Dial Transplant 2000; 15: 2029-2036. 